\title{
O FARMACÊUTICO NA ATENÇÃO PRIMÁRIA DO SUS
}

\author{
THE PHARMACIST IN THE PRIMARY CARE OF SUS
}

LENZI, Luana'; Garcia, Carolina G. ${ }^{2}$; PONTAROLO, Roberto ${ }^{3}$.

\section{1 - Mestre em Ciências Farmacêuticas, doutoranda do PPGCF/UFPR \\ 2 - Aluna de Graduação em Farmácia, UFPR \\ 3 - Professor do Departamento de Farmácia, UFPR}

\section{RESUMO:}

Com a criação do Sistema Único de Saúde (SUS), a assistência à saúde tornou-se disponível em todos os níveis de complexidade. Os medicamentos, um dos elementos mais utilizados para a melhoria da saúde, são também responsáveis por efeitos indesejados nos usuários, sendo algumas das causas o seu uso inadequado e a automedicação. Para promover a saúde e evitar problemas relacionados aos medicamentos a atenção farmacêutica é uma importante ferramenta e o farmacêutico um profissional imprescindível. Este trabalho traz uma reflexão sobre o papel do farmacêutico no SUS tendo como referência uma farmácia básica municipal no estado Paraná. Foram avaliados o perfil dos usuários, o número de atendimentos e a importância do farmacêutico neste processo. Os dados indicaram que o número de farmacêuticos presentes no SUS é insuficiente para atender a crescente demanda por medicamentos. Além disso, a formação acadêmica ainda é muito deficiente para que o profissional seja capaz de promover as transformações que a sociedade necessita e deseja.

Palavras-chave: Atenção farmacêutica; SUS; Medicamentos; Cuidados em Saúde.

\section{ABSTRACT:}

With the creation of the Unified Health System (SUS), health care became available at all levels of complexity. The drugs, one of the most used to improve health, are also responsible for undesirable effects on users, some of the causes of their inappropriate use and self-medication. To promote health and avoid problems related to drugs on pharmaceutical care is an important tool and essential to a professional pharmacist. This paper presents a reflection on the role of pharmacists in UHS pharmacy with reference to a municipal basic pharmacy in the state of Paraná. We evaluated the profile of users, the number of attendances and the importance of the pharmacist in this process. The data indicated that the number of pharmaceuticals present in the SUS is insufficient to meet the growing demand for drugs. In addition, academic training is still very poor so that the professional is able to promote the changes that society needs and 
wants.

Keywords: Pharmaceutical care; SUS; drugs; Health Care.

\section{INTRODUÇÃO}

Nas décadas de 80 e 90 o sistema de saúde brasileiro passou por transformações importantes com a criação e regulamentação do Sistema Único de Saúde (SUS). O SUS foi criado com a promulgação da Constituição Federal em 1988, permitindo ao Brasil institucionalizar o direito à saúde a todos os cidadãos brasileiros (BRASIL, 2007a; BRASIL, 2007b).

Os princípios fundamentais desse sistema caracterizam-se pela universalidade de acesso, integralidade da atenção, eqüidade, participação das comunidades e a descentralização. Engloba estabelecimentos públicos e o setor privado de prestação de serviços incluindo desde unidades de atenção básica até centros hospitalares de alta complexidade. A criação deste sistema de saúde representou uma nova forma de pensar, estruturar, desenvolver e produzir serviços e assistência em saúde, tanto para os gestores como para os trabalhadores e usuários do sistema (BRASIL, 2007a).

Dentre os fatores presentes na sociedade brasileira que promovem um aumento na demanda por medicamentos, estão o estímulo a automedicação e o uso desnecessário de medicamentos, fatos que determinam a necessidade da promoção do uso racional dos mesmos. Uma estratégia para a promoção do uso racional é a Atenção Farmacêutica, inserida no princípio da equidade do SUS (BRASIL, 2007c). Esta prática apresenta um componente fortemente humanístico, sendo focada diretamente no usuário e não nos medicamentos propriamente ditos, visando alcançar resultados específicos, como a melhoria da qualidade de vida (BRASIL, 2007a). Fundamentalmente, compreende ações preventivas que visam reduzir a morbimortalidade relacionada aos medicamentos, permitindo que recursos diretos (medicamentos, cuidados de profissionais de saúde) e indiretos (custo da internação, equipamentos de saúde etc.), que seriam utilizados em medidas curativas, sejam transferidos para o cuidado a outros usuários do SUS, como por exemplo, na atenção básica, melhorando assim a aplicação e o uso dos recursos (FELIPE, 2005).

A priorização de ações de caráter preventivo de promoção, proteção e recuperação da saúde tem permitido uma transformação no modelo de atenção prestada, proporcionado mudanças no quadro de morbimortalidade no país. O profissional farmacêutico é fundamental para viabilizar o planejamento de práticas focadas na atenção ao paciente na promoção do uso racional e na orientação para o sucesso da farmacoterapia [BRASIL, 2007ª; FELIPE, 2005).

O objetivo deste trabalho é contextualizar a atuação do farmacêutico no 
SUS e suas práticas na atenção primária à saúde por intermédio de exemplos práticos e atuais obtidos em uma farmácia básica municipal no Estado do Paraná.

\section{MÉTODOS}

Para a contextualização da importância da atuação profissional do farmacêutico no SUS realizou-se uma revisão bibliográfica por meio de consulta a bases de dados.

Para a realização da análise descritiva foram coletados dados relacionados ao perfil dos usuários e ao quantitativo de atendimentos da farmácia básica municipal de São José dos Pinhais, Paraná. Os dados foram extraídos do programa informatizado utilizado no município, chamado WinSaúde.

Utilizou-se a estatística descritiva, e as análises gráficas foram realizadas no programa Microsoft Office Excel 2007.

Este trabalho é fruto da etapa de caracterização do serviço de saúde no qual será desenvolvido um projeto maior, com duração prevista de 4 anos. Este projeto foi aprovado no Comitê de Ética em Pesquisa do Setor de ciências da Saúde da Universidade Federal do Paraná, sob o número de registro no SISNEP: CAAE 0036.0.091.000-11.

\section{RESULTADOS E DISCUSSÃO}

Em 1987 o governo federal propôs a Farmácia Básica, com a finalidade de racionalizar o fornecimento de medicamentos para a atenção primária em saúde. Contava com 48 medicamentos, todos constantes da Relação Nacional de Medicamentos Essenciais (Rename), destinando-se a atender as doenças prevalentes mais comuns, em especial no nível ambulatorial (COSENDEY, 2000). Posteriormente, foram acrescentados medicamentos de uso contínuo, totalizando 60 medicamentos considerados de maior demanda na rede pública de saúde (MEDICl et al., 1991; BERMUDEZ et al., 1995).

O Paraná foi o primeiro estado brasileiro a propor uma política de medicamentos destinada aos cuidados primários de saúde. Este fato ocorreu graças à priorização dada pelo governo do Estado à organização da Assistência Farmacêutica na rede de serviços públicos (GAF/SESAPR, 1995). Este programa de medicamentos denominou-se Programa Farmácia Básica (PFB-PR) e seguiu o modelo proposto em 1987, com algumas adequações e aprimoramentos. Os critérios utilizados na elaboração da relação dos medicamentos foram: os medicamentos deveriam constar da RENAME; serem eficazes, seguros e disponíveis para aquisição ao menor custo possível (COSENDEY et al., 2000). 
No município de São José dos Pinhais, no estado do Paraná, a farmácia Básica foi criada em 1996 para atender às necessidades básicas da população. O município, fundado em 08 de janeiro de 1853, possui $945,67 \mathrm{Km}^{2}$ de área territorial, sendo $80,1 \%$ área rural. Conforme dados obtidos do IBGE, em 2009, o município de São José dos Pinhais possuía 279.297 habitantes, com uma densidade demográfica de 287,28 hab/ $\mathrm{km}^{2}$. A Secretaria Municipal de Saúde dispõe de 35 Unidades Prestadoras de Serviço, sendo 22 Unidades Básicas e 13 Unidades Especializadas. Na área urbana estão localizadas todas as Unidades Especializadas e 12 das Unidades Básicas.

A Assistência Farmacêutica na atenção primária deve ser harmonizada com a demanda dos usuários a fim de garantir o acesso ao medicamento. Por este motivo, torna-se fundamental a padronização dos medicamentos, dos mecanismos de compra e a logística de distribuição, proporcionando maior racionalidade administrativa. Além disso, a padronização baseada em critérios epidemiológicos e farmacológicos evita as pressões mercadológicas e de relações interpessoais (MARIN et al., 2003; MESTRINER, 2003). Após muitos esforços, em 09 de agosto de 2010 foi publicada a primeira REMUME, contendo 203 medicamentos padronizados.

A prescrição e dispensação de medicamentos requerem qualificação permanente, pois sem dúvida, são as atividades que impactam diretamente sobre o uso racional dos medicamentos. A interação do profissional farmacêutico com o usuário deve ocorrer no momento da dispensação. Esse é o momento em que o usuário deve receber todas as informações e orientações sobre o correto uso do medicamento, objetivando alcançar melhoria da adesão ao tratamento e o sucesso na farmacoterapia.

A dispensação, representada pela relação direta com o usuário do medicamento, é enfatizada como a atividade mais importante do farmacêutico, pois este é o detentor privilegiado do conhecimento sobre o medicamento (HARDLING \& TAYLOR, 1997). Entretanto, em farmácias do SUS, geralmente o trabalho do farmacêutico está centrado na tecnologia de gestão do medicamento, no sentido de disponibilizar e garantir o acesso. Ao se adotar a conduta de que os medicamentos podem ser "entregues" por qualquer pessoa, valoriza-se mais a quantidade dos medicamentos "entregues" e não a qualidade e a resolutividade da assistência farmacêutica. Assim, tornam-se graves os problemas relacionados ao uso e à gestão inadequada dos medicamentos.

A dispensação de medicamentos foi definida na Política Nacional de Medicamentos como:

é o ato profissional farmacêutico de proporcionar um ou mais medicamentos a um paciente, geralmente, como resposta a apresentação de uma receita elaborada por um profissional autorizado. Neste ato o farmacêutico informa e orienta o paciente sobre o uso adequado do medicamento. São elementos importantes da orientação, entre outros, a ênfase no cumprimento da dosagem, a influência dos alimentos, a interação com outros medicamentos, o 
reconhecimento de reações adversas potenciais e as condições de conservação dos produtos (BRASIL, 2002).

Nesse conceito, a dispensação não se configura apenas como o fornecimento do medicamento prescrito, devendo atender a aspectos técnicos, com o objetivo de garantir a entrega do medicamento correto ao usuário, na dosagem e na quantidade prescrita, com instruções suficientes para seu uso adequado e guarda correta. Entre as orientações a serem repassadas, destaca-se a forma de administração, a possibilidade de ocorrência de efeitos colaterais e reações adversas, as interações com outros medicamentos e com alimentos, os cuidados sobre o armazenamento, entre outros. Desta forma, o usuário do medicamento terá as informações necessárias para seu uso seguro e correto podendo contribuir significativamente para o sucesso do tratamento (MSH, 1997).

Também é nesta etapa que devem ser observados aspectos relacionados à prescrição, entre eles a legibilidade da receita, que não deve deixar dúvidas ou dificuldades de interpretação. As normativas sobre prescrição versam que a mesma deve ser clara e legível, explicitando qual medicamento deve ser fornecido ao paciente, sua dose, posologia e modo como deve ser usado; não deve conter rasuras, trazer abreviaturas, códigos ou símbolos. Verificar, ainda, se foi emitida por profissional autorizado e se atende os aspectos legais, considerando que é um documento pelo qual se responsabilizam não só aqueles que prescrevem, mas também quem dispensa e administra os medicamentos nele registrados.

É importante que a dispensação seja precedida de uma boa acolhida do paciente, pois o mesmo, ao dirigir-se à farmácia, espera encontrar um profissional com conhecimentos técnicos que possa prestar informações adequadas sobre os medicamentos. O farmacêutico como integrante da equipe de saúde, deve dar os esclarecimentos necessários sobre a farmacoterapia, ofertando ao paciente suporte e apoio para o cumprimento do tratamento. A abordagem adequada do paciente na farmácia pode contribuir de forma decisiva para a adesão ou não ao tratamento proposto, e o farmacêutico deve aproveitar essa oportunidade para estabelecer uma relação de confiança com o usuário, devendo ouvi-lo, respeitá-lo e compreendê-lo.

Os dados levantados na farmácia básica municipal de São José dos Pinhais refletem o período de janeiro a agosto de 2011. Em média, são atendidos mensalmente $7.836,5( \pm 406,7)$ pacientes. $O$ acesso ao medicamento se dá mediante a apresentação da prescrição médica, do cartão do SUS e de um documento de identificação. A proporção de pacientes do gênero feminino foi maior em todos os meses avaliados, apresentando um percentual médio de $65,5 \%$ de todos os pacientes atendidos. A faixa etária dos usuários foi bem ampla, incluindo pacientes de 0 a 109 anos de idade. 0 quantitativo de pacientes atendidos a cada mês está representado no gráfico da 
figura 1.

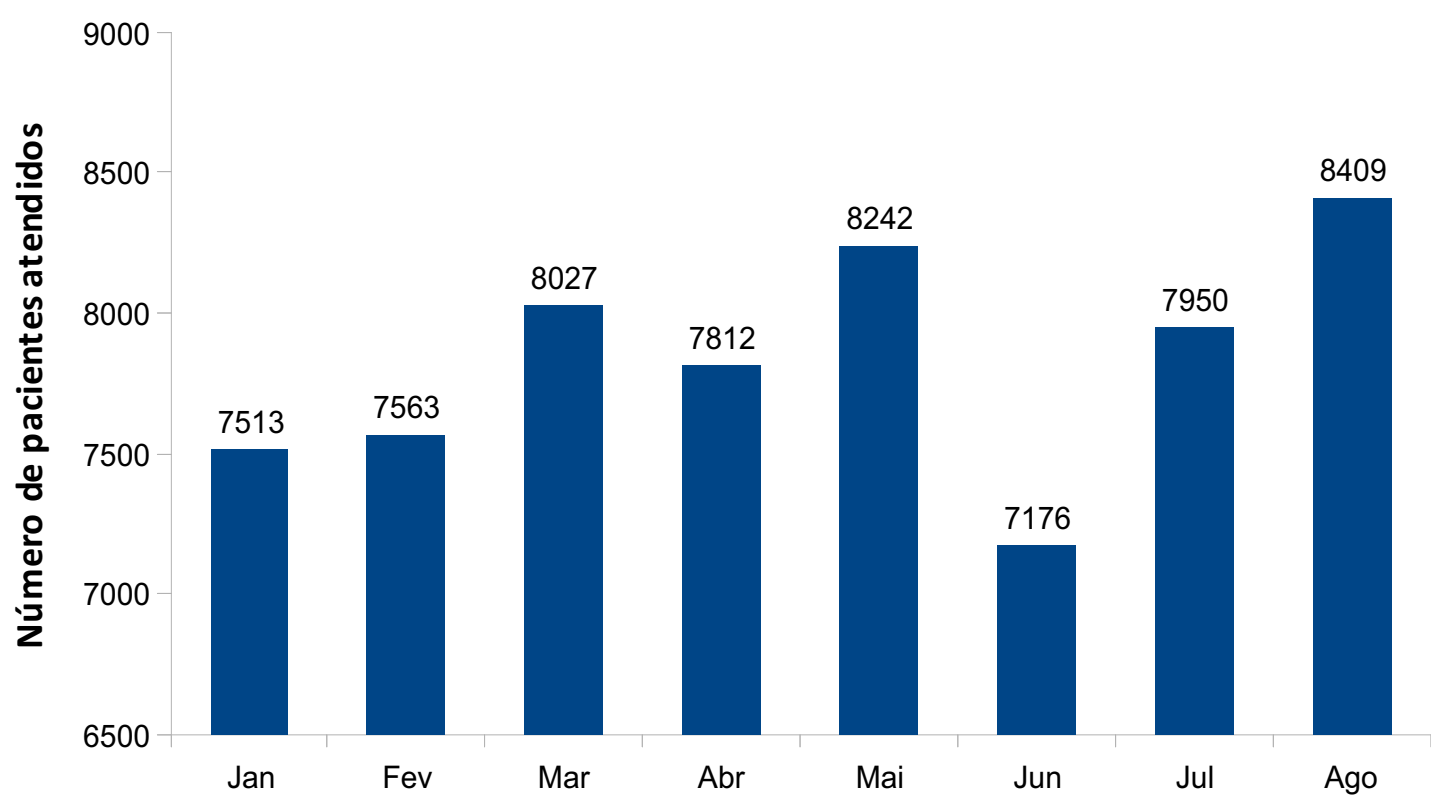

FIGURA 1 - Quantitativo mensal de pacientes atendidos de janeiro a agosto de 2011 na farmácia básica municipal de São José dos Pinhais, Paraná.

A farmácia conta com 3 farmacêuticas e 5 auxiliares administrativos. Na maioria das vezes, devido à grande demanda de pacientes e o pequeno número de profissionais de saúde, que muitas vezes estão envolvidos também com questões burocráticas importantes, o atendimento dos pacientes é feito pelos auxiliares administrativos com a supervisão dos farmacêuticos. Casos que requerem informações mais detalhadas acerca dos medicamentos ou pacientes que necessitam de maiores cuidados farmacoterapêuticos o atendimento e acompanhamento são feitos diretamente pelo farmacêutico, a fim de resolver e prevenir problemas reais e potenciais relacionados aos medicamentos. Por este motivo, a atuação do farmacêutico na atenção à saúde tem sido amplamente discutida e solicitada pela sociedade.

A definição inicial da atenção farmacêutica diz que essa prática "tem o objetivo de alcançar resultados que melhorem a qualidade de vida do paciente". Dessa forma, a profissão farmacêutica se move na direção do cuidado direto ao paciente e seu objeto social muda do medicamento para a pessoa. Entretanto, essa transição depende de um conhecimento mais voltado para as dimensões humanas da prática profissional, do processo saúde-doença e do uso do medicamento.

A implantação do acompanhamento farmacoterapêutico dos pacientes nas farmácias pode ser um meio de assegurar a qualificação e a humanização do atendimento ao usuário (OPAS, 2002). 
As diferentes iniciativas para que os medicamentos sejam prescritos e utilizados de forma correta, devem-se principalmente ao fato de que, apesar de serem importantes instrumentos de saúde, e quando utilizados de forma indevida, podem ser potenciais fontes de agravos e danos à saúde. Não é raro que erros de medicação e eventos adversos se constituam em causa de morte. Segundo dados da Organização Mundial de Saúde, mais de $50 \%$ de todos os medicamentos são prescritos, dispensados ou comercializados de forma inapropriada, sendo que metade dos usuários os utiliza incorretamente (OMS, 2011).

Para auxiliar na avaliação do perfil dos usuários, além da observação do número mensal de atendimentos, buscamos avaliar os principais medicamentos dispensados no período de janeiro a dezembro de 2011, cujo descritivo está representado na figura 2.

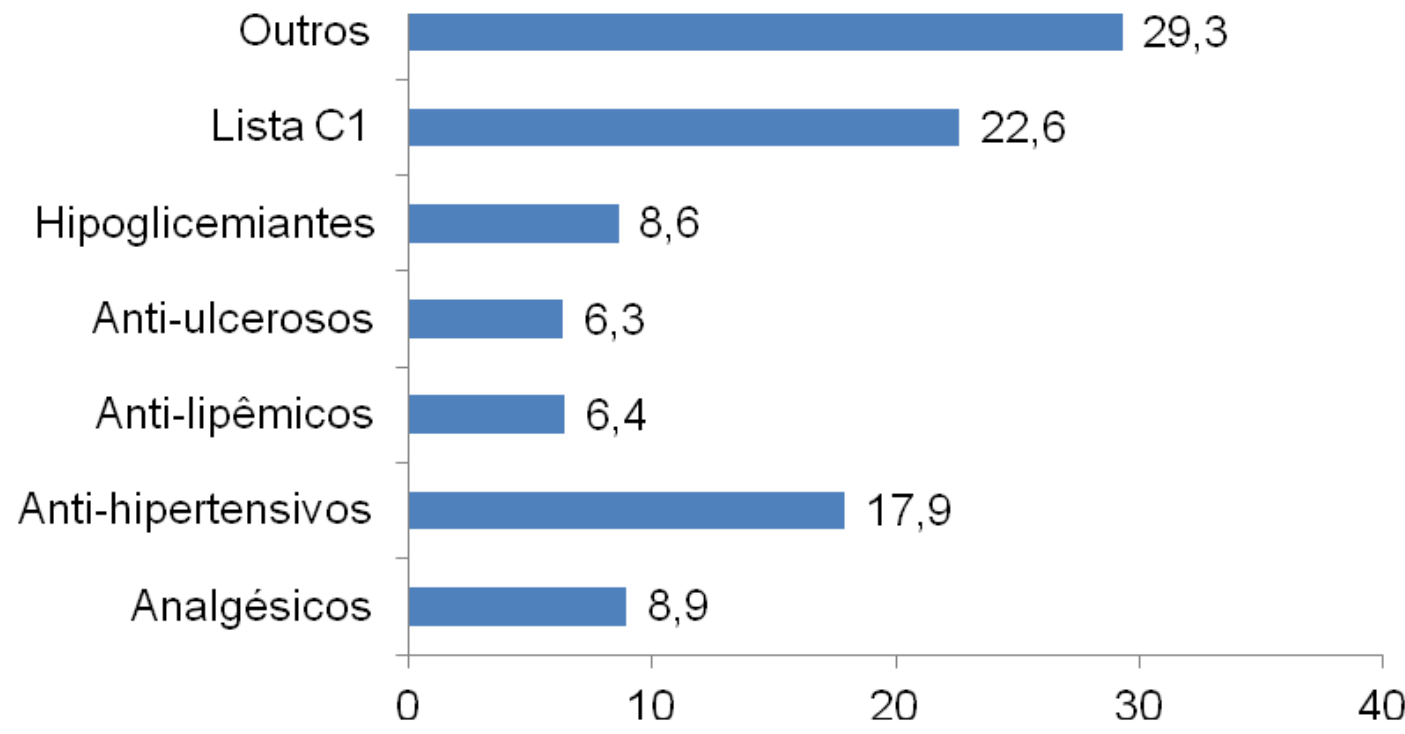

FIGU.... - . . .

2011 na farmácia básica municipal de São José dos Pinhais, Paraná

Esta análise indicou que a maior demanda ocorre por medicamentos para tratamento de diabetes, hipertensão e hiperlipidemia, totalizando $32,9 \%$ das dispensas mensais. Estas patologias são classificadas como doenças crônicas degenerativas que representam queda substancial na qualidade e na expectativa de vida. Por este motivo, torna-se imprescindível que o profissional de saúde possa orientar adequadamente estes indivíduos no que diz respeito ao correto uso da terapia, à adesão terapêutica e a adoção de medidas não farmacológicas que possam em conjunto, somar benefícios aos pacientes.

Os medicamentos da lista C1 da Portaria n 344/98 padronizados no município e incluídos no quantitativo ilustrado são: ácido valpróico , amitriptilina, biperideno, carba- 
mazepina, clorpromazina, fenitoína, haloperidol, imipramina, levomepromazina, lítio, nortriptilina e risperidona.

Vários são os obstáculos para o uso racional de medicamentos no Brasil: número excessivo de produtos farmacêuticos no mercado; prática da automedicação; falta de informações aos usuários; problemas nas prescrições (sobreprescrição, prescrição incorreta, prescrição múltipla, subprescrição etc.); disponibilidade ainda insuficiente de diretrizes clínicas tanto no setor privado como público; divulgação de informações inapropriadas sobre os medicamentos; propaganda e marketing de medicamentos, entre outros. Todos esses fatores, além de poderem trazer consequências graves para a saúde da população, levam a um dispêndio desnecessário de recursos financeiros, quer sejam eles originários de desembolso direto ou financiados com recursos públicos.

\section{CONCLUSÕES}

Para que possam funcionar adequadamente, todas as etapas relacionadas à Assistência Farmacêutica requerem, dentre outros, que o setor responsável pelas ações esteja estruturado, contando com profissionais qualificados e em número suficiente para para desenvolvê-las. Os resultados deste trabalho permitiram observar claramente que o número de profissionais farmacêuticos presentes nesta farmácia básica do SUS não é suficiente para atender corretamente à demanda da população que dela depende. Os órgãos públicos responsáveis devem perceber que a sociedade brasileira não pode mais conviver com o uso indiscriminado, não orientado e irracional dos medicamentos, que levam a prejuízos econômicos, sociais e humanos.

Consequentemente, as instituições de ensino que ministram cursos de farmácia devem privilegiar a formação de profissionais capazes de transformar o mercado, a partir do exercício de suas atividades como trabalhadores em saúde. A formação dos farmacêuticos deve ser realizada com estratégias educacionais que integrem o conhecimento teórico com a prática nos serviços de saúde. Entretanto, este é ainda um constante desafio, talvez o maior de todos os envolvidos no processo de construção da verdadeira Assistência à Saúde em nosso País.

As diretrizes curriculares dos cursos de graduação em farmácia ao instituírem o "farmacêutico generalista" dispõem que os currículos propostos possam construir perfil acadêmico e profissional com competências, habilidades e conteúdos, capazes de atuar com qualidade, eficiência e resolutividade, no Sistema Único de Saúde, considerado o processo da Reforma Sanitária Brasileira. Ao analisarmos o conteúdo das diretrizes curriculares de farmácia, identifica-se que os parâmetros sugeridos são amplos e genéricos, marcados pela possibilidade de variadas interpretações e sem 
garantia clara de componentes curriculares que assegurem a formação de farmacêuticos qualificados no âmbito do medicamento e da assistência farmacêutica.

\section{REFERÊNCIAS}

BRASIL. Ministério da Saúde. Secretaria de Políticas de Saúde. Departamento de

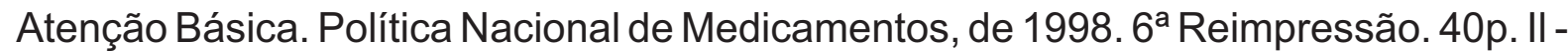
(Série C. Projetos, Programas e Relatórios, n. 25). Brasília: Ministério da Saúde, 2002a.

BRASIL. Ministério da Saúde. Secretaria de Ciência, Tecnologia e Insumos Estratégicos. Departamento de Assistência Farmacêutica e Insumos Estratégicos. O ensino e as pesquisas da atenção farmacêutica no âmbito do SUS / Ministério da Saúde, Secretaria de Ciência, Tecnologia e Insumos Estratégicos, Departamento de Assistência Farmacêutica e Insumos Estratégicos. - Brasília : Ministério da Saúde, 2007.

BRASIL. Conselho Nacional de Secretários de Saúde. Sistema Único de Saúde / Conselho Nacional de Secretários de Saúde. - Brasília : CONASS, 2007.

BRASIL. Conselho Nacional de Secretários de Saúde. Assistência Farmacêutica no SUS/ Conselho Nacional de Secretários de Saúde. - Brasília : CONASS, 2007.

BRASIL. Ministério da Saúde. Secretaria de Políticas de Saúde. Departamento de Atenção Básica. Política Nacional de Medicamentos, de 1998. $6^{a}$ Reimpressão. 40p. II (Série C. Projetos, Programas e Relatórios, n. 25). Brasília: Ministério da Saúde, 2002a.

COSENDEY, M.A.E. Análise da implantação do Programa Farmácia Básica: um estudo multicêntrico em cinco estados do Brasil. 2000. 358p. Tese (doutorado em Saúde Pública) - Escola Nacional de Saúde Pública Sérgio Arouca, Fundação Oswaldo Cruz, Rio de Janeiro.

COSENDEY, M. A. E.; BERMUDEZ, J. A. Z.; et al. Assistência farmacêutica na atenção básica de saúde: a experiência de três estados brasileiros Cad. Saúde Pública, Rio de Janeiro, 16(1):171-182, jan-mar, 2000.

FELIPE, J. S. Acesso aos medicamentos e primordial. Revista da Indústria Farmacêu- 
tica, [S.I.], n. 10, p. 30-5, 2005.

GAF/SESAPR (Grupo de Assistência Farmacêutica/Secretaria Estadual de Saúde do Paraná), 1995. Paraná Mais Saúde. Curitiba: Centro de Medicamentos do Paraná.

HARDLING, G.; TAYLOR, K. Responding to change; the case of community pharmacy in Great Britain. Soc Health \& IIIness 1997; 19 (5): 547-60.

MARIN, N.; LUIZA, V.L.; CASTRO, C.G.S.O.; SANTOS, S.M. Assistência farmacêutica para gerentes municipais. Rio de Janeiro: OPAS/OMS, 2003, 373p.

MEDICI, A. C.; OLIVEIRA, F.; BELTRÃO, K. I. A Política de Medicamentos no Brasil. Rio de Janeiro: Escola Nacional de Ciências Estatísticas/Instituto Brasileiro de Geografia e Estatística. 1991.

MESTRINER, D.C.P. O farmacêutico no serviço público de saúde: a experiência do município de Ribeirão Preto - SP [Dissertação] Ribeirão Preto: Faculdade de Medicina, USP; 2003.

MSH (Management Sciences for Health), 1997. Managing Drug Supply: The Selection, Procurement, Distribution, and Use of Pharmaceuticals. USA: Kumarian Press.

OMS. ORGANIZAÇÃO MUNDIAL DA SAÚDE. Medicines: rational use of medicines. $\mathrm{Fact} \mathrm{sh}$ e e t $\mathrm{n}$. $338, \mathrm{M}$ a y 2010 . D is ponível e m : $<$ http://www.who.int/mediacentre/factsheets/fs338/en/>. Acesso em 13 de agosto de 2011.

OPAS. Organização Panamericana de Saúde. Consenso brasileiro de atenção farmacêutica. Brasília: OPAS, 2002. 24 p. 